EESTI NSV TEADUSTE AKADEEMIA TOIMETISED. 28. KOIDË FOUSIKA * MATEMAATIKA. 1979, NR. 1

ИЗВЕСТИЯ АКАДЕМИИ НАУК ЭСТОНСКОИ ССР. ТОМ 28 ФИЗИКА * МАТЕМАТИКА. 1979, №2 1

Г. КАНГРО

\title{
СИЛЬНАЯ СУММИРУЕМОСТЬ ОРТОГОНАЛЬНЫХ РЯДОВ СО СКОРОСТЬЮ *
}

\author{
(Представлена А. Хумалом)
}

1. Пусть ** $A=\left(a_{n k}\right)$ и $\lambda=\left(\lambda_{n}\right)$, где $0<\lambda_{n} \uparrow$. Последовательность $x=\left(\xi_{k}\right)$ называется регулярно $A^{\lambda}$-суммируемой к $\xi$, если

$$
\lim _{n} \lambda_{n}\left(\sum_{k} a_{n k} \xi_{k}-\xi\right)=0,
$$

и сильно $A^{\lambda}$-суммируемой в степени $r>0$ (коротко $\left[A^{\lambda}\right]_{r}$-суммируемой) к $\xi$, если

$$
\lim _{n} \lambda_{n}^{r} \sum_{k}\left|a_{n k}\right|\left|\xi_{k}-\xi\right|^{r}=0 .
$$

При $\lambda_{n}=O(1)$ (напр., при $\lambda_{n}=1$ ) $A^{\lambda}$-суммируемость переходит в $A$-суммируемость, а $\left[A^{\lambda}\right]_{r}$-суммируемость - в $[A]_{r}$-суммируемость. Отметим, что при $0<r^{\prime}<r$ из [ $\left.A^{\lambda}\right]_{r}$-суммируемости последовательности

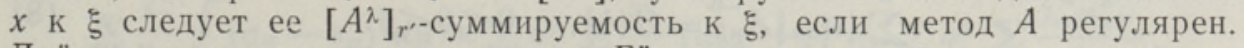
Действительно, в силу неравенства Гёльдера имеем

$$
\lambda_{n}^{r^{\prime}} \sum_{k}\left|a_{n k}\right|\left|\xi_{k}-\xi\right|^{r^{\prime}} \leqslant\left(\sum_{k}\left|a_{n k}\right|^{r /\left(r-r^{\prime}\right)}\right)^{1-r^{\prime} / r}\left(\lambda_{n}^{r} \sum_{k}\left|a_{n k}\right|\left|\xi_{k}-\xi\right| r\right)^{r^{\prime} / r},
$$

откуда ввиду регулярности метода $A$, неравенства $r /\left(r-r^{\prime}\right)>1$ и ра-

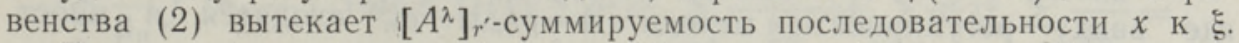

Если $a_{n 0}+a_{n 1}+\ldots=1$, то из (2) при $r \geqslant 1$ следует (1), т. е. из $\left[A^{\lambda}\right]_{r}$-суммируемости последовательности $x$ при $r \geqslant 1$ следует ее $A^{\lambda}$-суммируемость к тому же значению.

Рассмотрим ортогональный ряд

$$
f=\sum a_{n} \varphi_{n}
$$

с $\Sigma a_{n}{ }^{2}<\infty$, где $\left(\varphi_{n}\right)$ - система действительных функций, ортонормальная на заданном множестве $X$ по некоторой положительной $\sigma$-аддитивной мере $\mu$, а $f-$ вещественно-значная функция, определенная почти всюду (п. в.) на $X$, к которой ряд $\Sigma a_{n} \varphi_{n}$ сходится в пространстве

* Это посмертная статья $Г$. Кангро, подготовленная на основе оставшихся заметок к печати его учениками С. Бароном и Э. Юримяэ.

** Свободные индексы и индексы суммирования пробегают все целочисленные значения $0,1,2, \ldots$. 
$L_{\mu}^{2}$ (согласно теореме Рисса-Фишера). Возникает следующая проблема: при каких условиях, наложенных на метод $A$ и скорость суммируемости $\lambda$, из $A^{\lambda}$-суммируемости п. в. ряда (3) следует его $\left[A^{\lambda}\right]_{r}$-суммируемость п. в.?

Поставленная проблема рассматривалась в [1 $\left.{ }^{1}\right]$ для метода ВаллеПуссена, в $\left[{ }^{2}\right]$ для метода Чезаро $(C, \alpha)$ и в $\left[{ }^{3}\right]$ для методов класса $A^{p}$. Ниже доказывается общая теорема, из которой можно вывести результаты работ $\left[{ }^{1-3}\right]$, а также многие результаты, относящиеся к сильной суммируемости без скорости (т. е. к случаю $\left.\lambda_{n}=1\right)$. Предварительно докажем несколько лемм.

2. Для регуля рного метода суммирования $A=\left(a_{n k}\right)$ введем обозначения

$$
A_{n k}=\sum_{v=0}^{k}\left|a_{n v}\right|, \quad \alpha_{n k}=\sum_{v=k}^{\infty} a_{n v} .
$$

В дальнейшем предполагается выполнение условия

$$
\sum_{k} a_{n k}=1
$$

которое имеет место для всех практически важных методов суммирования. Из (5) в силу (4) вытекает

$$
\left|1-\alpha_{n k}\right| \leqslant A_{n k},
$$

причем ввиду регулярности метода $A$ имеем

$$
A_{n k}=O(1) .
$$

При $0 \leqslant \theta<1$ справедливо неравенство (см. [ $\left.{ }^{4}\right]$, с. 5 )

$$
\left|a_{n k}\right| A_{n k}^{-\theta} \leqslant \frac{1}{1-\theta}\left(A_{n k}^{1-\theta}-A_{n, k-1}^{1-\theta}\right), \quad k \geqslant x,
$$

где $x=x(n)$ - наименьшее значение индекса $k$, при котором $a_{n k} \neq 0$ (или $A_{n k} \neq 0$ ). Отсюда в силу (7) следует

$$
\sum_{k=x}^{n}\left|a_{n k}\right| A_{n k}^{-\theta}=O(1), \quad 0 \leqslant \theta<1 .
$$

Наконец, обозначим

$$
s_{k}=\sum_{v \leqslant k} a_{v} \varphi_{v}, \quad \sigma_{n}=\sum_{k} a_{n k} s_{k},
$$

где ввиду регулярности метода $A$ ряды $\sigma_{n}$ сходятся в пространстве $L^{2} \mu$.

Л е м а 1. Из условий***

$$
\begin{aligned}
& 1^{\circ} \sum_{k} a_{k}^{2} \lambda_{k}^{2}<\infty, \\
& 2^{\circ} \lambda_{n} A_{n k}^{\theta}=O\left(\lambda_{k}\right) \quad \text { при некотором } 0 \leqslant \theta<1, \\
& 3^{\circ} \sum_{n \geqslant k} \frac{A_{n k}^{2(1-\theta)}}{\beta_{n}}=O(1),
\end{aligned}
$$

*** Условие $2^{\circ}$ при $\theta \in(0,1)$ достаточно (а при некоторых ограничениях на величины $A_{n k}$ и необходимо) для того, чтобы треугольный регулярный метод $A$ сохранял $\lambda$-ограниченность (см. $\left[{ }^{4}\right]$, с. 5$)$. 


$$
4^{\circ} \sum_{n<k} \frac{\alpha_{n k}^{2}}{\beta_{n}}=O(1)
$$

где $\beta_{n}$ - некоторые положительные числа, вытекает

$$
\int \sum_{n} \frac{\lambda_{n}^{2}}{\beta_{n}}\left(\sigma_{n}-s_{n}\right)^{2} d \mu=O(1) \sum_{k} a_{k}^{2} \lambda_{k}^{2} .
$$

Д ок а з а тельст в о. Применяя преобразование Абеля

$$
\sum_{k=0}^{m} \alpha_{n k} a_{k} \varphi_{k}=\sum_{k=0}^{m-1} a_{n k} s_{k}+\alpha_{n m} s_{m},
$$

в силу регулярности метода $A$ и сходимости последовательности $\left(s_{k}\right)$ в $L^{2} \mu$ получаем

$$
\sum_{k} \alpha_{n k} a_{k} \varphi_{k}=\sum_{k} a_{n k} s_{k},
$$

где ряд в левой части равенства сходится в $L^{2} \mu$. Следовательно,

$$
\begin{aligned}
\sigma_{n}-s_{n} & =\sum_{k} \alpha_{n k} a_{k} \varphi_{k}-\sum_{k \leqslant n} a_{k} \varphi_{k}= \\
& =\sum_{k \leqslant n}\left(\alpha_{n k}-1\right) a_{k} \varphi_{k}+\sum_{k>n} \alpha_{n k} a_{k} \varphi_{k} .
\end{aligned}
$$

После почленного интегрирования, допустимого ввиду слабой сходимости последнего ряда в $L_{\mu}^{2}$, находим

$$
\int\left(\sigma_{n}-s_{n}\right)^{2} d \mu=\sum_{k \leqslant n}\left(\alpha_{n k}-1\right)^{2} a_{k}^{2}+\sum_{k>n} \alpha_{n k}^{2} a_{k}^{2}
$$

откуда после умножения на $\left(\lambda_{n} / \beta_{n}\right)^{2}$ и замены порядка суммирования получаем

$$
\sum_{n} \frac{\lambda_{n}^{2}}{\beta_{n}} \int\left(\sigma_{n}-s_{n}\right)^{2} d \mu=\sum_{k} a_{k}^{2} \sum_{n \geqslant k} \frac{\lambda_{n}^{2}}{\beta_{n}}\left(\alpha_{n k}-1\right)^{2}+\sum_{k>0} a_{k}^{2} \sum_{n<k} \frac{\lambda_{n}^{2} \alpha_{n k}^{2}}{\beta_{n}},
$$

или, имея в виду (6) и условия $2^{\circ}-4^{\circ},-$

$$
\begin{aligned}
\sum_{n} \frac{\lambda_{n}^{2}}{\beta_{n}} \int\left(\sigma_{n}-s_{n}\right)^{2} d \mu & \leqslant O(1) \sum_{k} a_{k}^{2} \lambda_{k}^{2} \sum_{n \geqslant k} \frac{A_{n k}^{2(1-\theta)}}{\beta_{n}}+\sum_{k>0} a_{k}^{2} \lambda_{k}^{2} \sum_{n<k} \frac{\alpha_{n k}^{2}}{\beta_{n}}= \\
& =O(1) \sum_{k} a_{k}^{2} \lambda_{k}^{2} .
\end{aligned}
$$

Применяя лемму Фату к последовательности

$$
\left(\sum_{k \leqslant n} \frac{\lambda_{k}^{2}}{\beta_{k}}\left(\sigma_{k}-s_{k}\right)^{2}\right)
$$

в силу условия $1^{\circ}$ получаем (9).

Л е м м 2. Из условий $1^{\circ}-4^{\circ}$ леммь 1 и условия

$$
5^{\circ} \quad a_{n k} \beta_{k}=O(1) A_{n k}^{\beta}, \quad 0<\beta \leqslant 1,
$$


следует

$$
\int\left(\sup _{n} \lambda_{n}^{r} \sum_{k}\left|a_{n k}\right|\left|\sigma_{k}-s_{k}\right|^{r}\right)^{2 / r} d \mu=O(1) \sum_{k} a_{k}^{2} \lambda_{k}^{2}
$$

при $0<r \leqslant 2, r<\beta \theta^{-1}$.

Доказательство. Рассмотрим отдельно случаи $r<2$ и $r=2$.

1) Если $r<2$, то, применяя к ряду

$$
\sum_{k}\left|a_{n k}\right|\left|\sigma_{k}-s_{k}\right|^{r}=\sum_{k} \frac{\left|a_{n k}\right| \beta_{k}^{r / 2}}{\lambda_{k}^{r}} \cdot \frac{\lambda_{k}^{r}\left|\sigma_{k}-s_{k}\right|^{r}}{\beta_{k}^{r / 2}}
$$

неравенство Гёльдера при $s=2 /(2-r)$ и $t=2 / r$, получаем

$$
\sum_{k}\left|a_{n k}\right|\left|\sigma_{k}-s_{k}\right|^{r} \leqslant\left(\sum_{k} \frac{\left|a_{n k}\right|^{s} \beta_{k}^{u}}{\lambda_{k}^{2 u}}\right)^{1 / s}\left(\sum_{k} \frac{\lambda_{k}^{2}\left|\sigma_{k}-s_{k}\right|^{2}}{\beta_{k}}\right)^{1 / t},
$$

где $u=r s / 2=s-1$. Из условий $2^{\circ}, 5^{\circ}$ и $\lambda_{n} \uparrow$ следует

$$
\begin{aligned}
\lambda_{n}^{r}\left(\sum_{k} \frac{\left|a_{n k}\right|^{s} \beta_{k}^{u}}{\lambda_{k}^{2 u}}\right)^{1 / s} & =\left[\lambda_{n}^{2 u} \sum_{k} \frac{\left|a_{n k}\right|\left(\left|a_{n k}\right| \beta_{k}\right)^{u}}{\lambda_{k}^{2 u}}\right]^{1 / s}= \\
& =O(1)\left[\sum_{k=x}^{n}\left|a_{n k}\right| A_{n k}^{u(\beta-2 \theta)}+\sum_{k>n}\left|a_{n k}\right| A_{n k}^{\beta u}\right]^{1 / s}
\end{aligned}
$$

где $x=\varkappa(n)$ - наименьшее значение индекса $k$, при котором $a_{n k} \neq 0$. В квадратных скобках обе суммы ограничены при $\theta \leqslant \beta / 2$ ввиду (7), а при $\theta>\beta / 2-$ ввиду (8), так как (в силу неравенства $\theta<\beta / r$ )

$$
0<u(2 \theta-\beta)<\frac{r}{2-r}\left(\frac{2 \beta}{r}-\beta\right)=\beta \leqslant 1
$$

Тем самым из (11) следует

$$
\lambda_{n}^{r} \sum_{k}\left|a_{n k}\right|\left|\sigma_{k}-s_{k}\right|^{r}=O(1)\left(\sum_{k} \frac{\lambda_{k}^{2}\left|\sigma_{k}-s_{k}\right|^{2}}{\beta_{k}}\right)^{r / 2},
$$

откуда при помощи леммы 1 и получается (10).

2) Покажем, что условие (12) выполнено и при $r=2$. Действительно, поскольку при $r=2$ имеет место неравенство $\theta<\beta / 2$, то из условий $5^{\circ}$ и $2^{\circ}$ вытекает

$$
\frac{\lambda_{n}^{2}\left|a_{n k}\right| \beta_{k}}{\lambda_{k}^{2}}=O(1)\left(\frac{\lambda_{n}}{\lambda_{k}} A_{n k}^{\beta / 2}\right)^{2}=O(1)
$$

Следовательно,

$$
\begin{aligned}
\lambda_{n}^{2} \sum_{k}\left|a_{n k}\right|\left|\sigma_{k}-s_{k}\right|^{2} & =\sum_{k} \frac{\lambda_{n}^{2}\left|a_{n k}\right| \beta_{k}}{\lambda_{k}^{2}} \cdot \frac{\lambda_{k}^{2}\left|\sigma_{k}-s_{k}\right|^{2}}{\beta_{k}}= \\
& =O(1) \sum_{k} \frac{\lambda_{k}^{2}\left|\sigma_{k}-s_{k}\right|^{2}}{\beta_{k}},
\end{aligned}
$$


что и доказывает справедливость соотношения (12), а вместе с тем и соотношения (10) - также при $r=2$.

Лемма доказана.

Примечание 1 . Из доказательства леммы 2 следует, что если $A=\left(a_{n k}\right)$ - верхняя треугольная матрица (т. е. если $a_{n k}=0$ при $k<n)$, то условне $5^{\circ}$ в лемме 2 можно заменить более слабым условием $a_{n k} \beta_{k}=O(1)$.

Л е м м а 3. Пусть **** $\lambda_{n} \neq O(1)$. Если метод $A$ сохраняет ограниченность ***** и выполнено условие $2^{\circ}$ леммы 1 , то при $0<p<\beta \theta^{-1}$ матрица

$$
\left(\lambda_{n}^{p}\left|a_{n k}\right| \lambda_{k}^{-p}\right)
$$

сохраняет класс нуль-последовательностей.

Д ок а з а тел ь с в о. Нам надо доказать, что из условий леммы 3 вытекают условия (см. $\left[{ }^{10}\right]$, с. 17 )

$$
\lim _{n} \lambda_{n}^{p} a_{n k}=0, \quad \lambda_{n}^{p} \sum_{k}\left|a_{n k}\right| / \lambda_{k}^{p}=O(1) .
$$

Пусть число $s$ удовлетворяет условию

$$
p<s<\beta \theta^{-1} .
$$

Из условия $2^{\circ}$ леммы 1 получаем

$$
\lambda_{n}^{s} A_{n k}^{\theta s}=O(1) \lambda_{k}^{s}
$$

где $0 \leqslant \theta s<1$, ибо $\beta \leqslant 1$. Поскольку выполнено условие (7), то согласно (8) имеем

$$
\lambda_{n}^{s} \sum_{k=x}^{n} \frac{\left|a_{n k}\right|}{\lambda_{k}^{s}}=O(1) \sum_{k=\kappa}^{n} \frac{\left|a_{n k}\right|}{A_{n k}^{\theta s}}=O(1),
$$

откуда ввиду $p<s$ и $\lambda_{n} \uparrow \infty$ (и, значит, $\left.\lambda_{n}{ }^{p-s} \rightarrow 0\right)$ пр.і гомощи (7) следуют условия (13).

Лемма доказана.

Л емм а 4 . Из условий $1^{\circ}-4^{\circ}$ леммь 1 и условия $5^{\circ}$ леммь 2 с $\beta=1$ вытекает

$$
\lim _{n} \lambda_{n}^{r} \sum_{k}\left|a_{n k}\right|\left|\sigma_{k}-s_{k}\right|^{r}=0 \quad \text { n. } 8 .
$$

при $0<r \leqslant 2, r<\theta^{-1}$.

Доказательство. Ввиду условия $1^{\circ}$ соответственно каждому числу $\varepsilon>0$ найдется натуральное число $N$ такое, что

$$
\sum_{k>N} \lambda_{k}^{2} a_{h}^{2}<\varepsilon^{3}
$$

Обозначив

$$
b_{k}=\left\{\begin{array}{ll}
a_{k}, & k \leqslant N, \\
0, & k>N,
\end{array} \quad c_{k}= \begin{cases}0, & k \leqslant N, \\
a_{k}, & k>N,\end{cases}\right.
$$

находим, что $a_{k}=b_{k}+c_{k}$ и, следовательно, лярен.

**** Если $\lambda_{n}=O(1)$, то утверждение леммы имеет место, когда метод $A$ регу-

***** Следовательно (cм. $\left[{ }^{10}\right]$, теорема 2.2), метод $A$ удовлетворяет условию (7). 


$$
s_{k}=s_{k}(b)+s_{k}(c), \quad \sigma_{n}=\sigma_{n}(b)+\sigma_{n}(c),
$$

где

$$
s_{k}(b)=\sum_{v \leqslant k} b_{v} \varphi_{v}, \quad \sigma_{n}(b)=\sum_{k} a_{n k} s_{k}(b) .
$$

В силу неравенства $|u+v|^{r} \leqslant 2^{r}\left(|u|^{r}+|v|^{r}\right)$ имеем

$$
\lambda_{n}^{r} \sum_{k}\left|a_{n k}\right|\left|\sigma_{k}-s_{k}\right|^{r} \leqslant 2^{r}\left[A_{n}(b)+A_{n}(c)\right] \text {, }
$$

где

$$
A_{n}(b)=\lambda_{n}^{r} \sum_{k}\left|a_{n k}\right|\left|\sigma_{k}(b)-s_{k}(b)\right|^{r} .
$$

Достаточно показать, что

$$
\lim _{n} A_{n}(b)=0, \quad \lim _{n} A_{n}(c)=0 \quad \text { п. в. }
$$

При $k>N$ в силу (5) и определения $b_{k}$ находим

$$
\begin{aligned}
\sigma_{k}(b)-s_{k}(b) & =\sum_{v \leqslant N} a_{k v} s_{v}(b)+\sum_{v>N} a_{k v} s_{N}(b)-s_{N}(b)= \\
& =\sum_{v \leqslant N} a_{k v}\left[s_{v}(b)-s_{N}(b)\right],
\end{aligned}
$$

откуда при помощи первого из условий (13), взяв в нем $p=1$, ввиду $\theta^{-1}>1$ получаем

$$
\lim _{k} \lambda_{k}\left[\sigma_{k}(b)-s_{k}(b)\right]=0 .
$$

Отсюда на основе леммы 3 следует

$$
\lim _{n} A_{n}(b)=0
$$

Из условий $1^{\circ}-5^{\circ}$ согласно лемме 2 и условию (15) вытекает

$$
\int\left[\sup _{n} A_{n}^{1 / r}(c)\right]^{2} d \mu \leqslant M \sum_{k>N} a_{k}^{2} \lambda_{k}^{2}<M \varepsilon^{3}
$$

при $0<r \leqslant 2, r<\theta^{-1}$, где $M-$ некоторая постоянная. Из (18) следует

$$
\mu\left\{t: \varlimsup_{n} A_{n}^{1 / r}(c)>\varepsilon\right\} \leqslant M \varepsilon .
$$

Действительно, если бы имело место неравенство

$$
\mu\left\{t: \varlimsup_{\lim } A_{n}^{1 / r}(c)>\varepsilon\right\}>M \varepsilon,
$$

то тем более

$$
\left.\mu\left\{t:\left[\sup _{n} A_{n}^{1 / r}(c)\right]^{2}>\varepsilon^{2}\right]\right\}>M \varepsilon
$$

и, следовательно,

$$
\int\left[\sup _{n} A_{n}^{1 / r}(c)\right]^{2} d \mu>M \varepsilon^{3},
$$

что противоречит неравенству (18), 
При произвольном фиксированном $\delta>0$ имеем

$$
\left\{t: \varlimsup_{n} A_{n}^{1 / r}(c)>0\right\} \subset \underset{k \geqslant 1}{\bigcup}\left\{t: \varlimsup_{n} A_{n}^{1 / r}(c)>\frac{\delta}{2^{k}}\right\},
$$

откуда ввиду (19)

$$
\mu\left\{t: \varlimsup_{n} A_{n}^{1 / r}(c)>0\right\} \leqslant \sum_{k \geqslant 1} M \frac{\delta}{2^{k}}=M \delta
$$

и при $\delta \rightarrow 0$

$$
\lim _{n} A_{n}(c)=0 \text { п. в. }
$$

Из (16) в силу (17) и (20) следует (14).

Лемма доказана.

П р и меч ание 2 . Если $A=\left(a_{n k}\right)$ - верхняя треугольная матрица, то ввиду примечания 1 условие $5^{\circ}$ можно заменить более слабым условием $a_{n k} \beta_{k}=O(1)$.

3. Следующая теорема дает ответ на поставленную в п. 1 проблему.

Т е о рем а. Если регулярный метод суммирования $A=\left(a_{n k}\right)$ при условии (5) и скорость $\lambda=\left(\lambda_{n}\right)$ с $0<\lambda_{n} \uparrow$ удовлетворяют условиям $1^{\mathrm{q}-5^{\circ}}$, то из регулярной $A^{\lambda}$-суммируемости п. в. ряда (3) к функции $f$ следует его $\left[A^{\lambda}\right]_{r}$-суммируемость п. в. $\kappa f$, если $0<r \leqslant 2, r<\theta^{-1}$.

Д ок а з а т льство. Имеем

$$
\lambda_{n}^{r} \sum_{k}\left|a_{n k}\right|\left|s_{k}-f\right|^{r} \leqslant 2^{r} \lambda_{n}^{r} \sum_{k}\left|a_{n k}\right|\left|s_{k}-\sigma_{k}\right|^{r}+2^{r} \lambda_{n}^{r} \sum_{k}\left|a_{n k}\right|\left|\sigma_{k}-f\right|^{r},
$$

где первый член в правой части неравенства стремится к нулю п. в. согласно лемме 4, а второй член - согласно лемме 3, поскольку $\lambda_{k}\left|\sigma_{k}-f\right|$, по предположению, стремится к нулю п. в.

При $\lambda_{n}=1$ из теоремы при $\theta=0$ получается следующее следствие (относительно условия в) см. примечания 1 и 2).

Следствие. Если регулярный метод суммирования $A=\left(a_{n k}\right)$ при условии (5) удовлетворяет условиям
a) $\sum_{n \geqslant k} \frac{A_{n k}^{2}}{\beta_{n}}=O(1)$,
б) $\sum_{n<k} \frac{\alpha_{n k}^{2}}{\beta_{n}}=O(1)$,
в) $a_{n k} \beta_{k}=O(1)$

при некоторых $\beta_{n}>0$, то из А-суммируемости п. в. ряда (3) $с \Sigma a_{k}^{2}<$ $<\infty \kappa$ функции $f$ следует есо [A] $]_{r}$-суммируемость п. в. $\kappa f$, если $0<r \leqslant 2$.

Следствие содержит известные результаты для методов арифметических средних $\left[{ }^{5,6}\right]$, взвешенных средних Рисса $\left(R, p_{n}\right)\left[{ }^{7,8}\right]$, ВаллеПуссена ***** $\left(V, \alpha_{n}\right)\left[{ }^{9}\right]$. Выполнение условий a)-в) проверяется просто, если положить $\beta_{n}=n+1, P_{n} / p_{n}, \alpha_{n}$ соответственно.

****** Оказывается, что для метода арифметических средних и метода ВаллеПуссена имеет место даже очень сильная суммируемость. 
ЛИ Т Е Р А Т У Р А

1. Alexts, G., Králik, D., Acta math. Acad. sci. hung., 16, № 1-2, 43-49 (1965).

2. Sunouchi, G., Indian J. Math., 9, № 1, 237-246 (1967).

3. Болгов В. А., Ефим о в А. В., Изв. АН СССР, Сер. матем., 35, № 6, 1389 $1408(1971)$.

4. К а н г ро Г., Изв. АН ЭССР, Физ. Матем., 23, № 1, 3-11 (1974).

5. Z y g m un d, A., Fund. math., 10, 356-362 (1926).

6. T a nd or i, K., Acta sci. math., 19, 18-25 (1958).

7. Me de r, J., Stud. math., 20, № 3, 285-300 (1961)

8. Le i nd l e r, L., Acta math. Acad. sci. hung., 13, № 3-4, 401-414 (1962).

9. Le i nd l e r, L., Acta math. Acad. sci. hung., 16, № 3-4, 375-387 (1965).

10. Бар он С. А., Введение в теорию суммируемости рядов, Таллин, «Валгус», 1977.

Тартуский государственныи университет
Поступила в редакцию 9/III 1978

G. KANGRO

\section{ORTOGONAALRIDADE TUGEV SUMMEERUVUS KIIRUSEGA}

Olgu $A=\left(a_{n k}\right)$ ja $\lambda=\left(\lambda_{k}\right)$, kus $0<\lambda_{k} \uparrow$. Jada $x=\left(\xi_{k}\right)$ nimetatakse regulaarselt $A^{\lambda}$-summeeruvaks arvuks $\xi$, kui $x$ täidab tingimust (1) ning astmega $r>0$ tugevalt $A^{\lambda}$-summeeruvaks (lühidalt $\left[A^{\lambda}\right]_{r}$-summeeruvaks) arvuks $\xi$, kui $x$ rahuldab tingimust (2). On tõestatud, et kui seost (5) rahuldava regulaarse menetluse $A$ ja kiiruse $\lambda$ puhul on täidetud tingimused $1^{\circ}-5^{\circ}$, siis reaalse ortogonaalrea (3) regulaarsest $A^{\lambda}$-summeeruvusest peaaegu kõikjal funktsiooniks $f$ järeldub selle rea $\left[A^{\lambda}\right]_{r}$-summeeruvus samaks funktsiooniks peaaegu kõikjal $r \leqslant 2, r<\theta^{-1}$ korral.

G. KANGRO

\section{THE STRONG SUMMABILITY OF ORTHOGONAL SERIES WITH SPEED}

Let $A=\left(a_{n k}\right)$ and $\lambda=\left(\lambda_{k}\right)$, where $0<\lambda_{k} \uparrow$. The sequence $x=\left(\xi_{k}\right)$ is called regularly $A^{\lambda}$-summable to $\xi$ if $x$ satisfies the condition (1). The sequence $x$ is called strongly $A^{\lambda}$-summable (briefly $\left[A^{\lambda}\right]_{r}$-summable) to $\xi$ with the degree $r>0$, if for $x$ the condition (2) is fulfilled.

It is proved: If $A$ is a regular method with (5) and $1^{\circ}-5^{\circ}$ are satisfied, then the real orthogonal series (3) is almost everywhere $\left[A^{\lambda}\right]_{r}$-summable to the function $f$ for $r \leqslant 2$, $r<\theta^{-1}$, if (3) is regularly $A^{\lambda}$-summable almost everywhere to the same $f$. 